\title{
New Concepts and Perspectives on Micro-Rotorcraft and Small Autonomous Rotary-Wing Vehicles
}

\author{
L.A. Young \\ E.W. Aiken \\ Army/NASA Rotorcraft Division \\ NASA Ames Research Center \\ Moffett Field, CA 94035 \\ J.L. Johnson \\ Aerospace Computing, Inc. \\ Los Gatos, CA \\ R. Demblewski \\ College of San Mateo \\ NASA Education Associates Program \\ J. Andrews \\ Massachusetts Institute of Technology \\ NASA Undergraduate Student Research Program
}

\section{Introduction}

A key part of the strategic vision for rotorcraft research as identified by senior technologists within the Army/NASA Rotorcraft Division at NASA Ames Research Center is the development and use of small autonomous rotorcraft (Ref. 1). Small autonomous rotorcraft are defined for the purposes of this paper to be a class of vehicles that range in size from rotary-wing micro air vehicles (MAVs) to larger, more conventionally sized, rotorcraft uninhabited aerial vehicles (UAVs) - i.e. vehicle gross weights ranging from hundreds of grams to thousands of kilograms.

To be presented at the $20^{\text {th }}$ AIAA Applied Aerodynamics Conference, St. Louis, MO, June 24-27, 2002.
Development of small autonomous rotorcraft represent both a technology challenge and a potential new vehicle class that will have substantial societal impact:

National Security --

The global urbanization of the late twentieth and early twenty-first century poses unique challenges for maintaining peace and security throughout the world. Lessons from Somalia, Bosnia, and Kosovo, clearly show the necessity for updating military doctrine and mechanization to account for warfighting in the urban environment. A potential innovative 
solution for both these military and public service problems is the development, demonstration, and use of autonomous rotorcraft UAV for urban environment missions.

Personal Transport --

The future mobility requirements for the traveling public will dictate the development of rotorcraft and other vertical lift aerial vehicles for personal transport. Key to the development of a personal transport vertical lift aerial vehicle is the ease, and safety, of flight. Ideally, one would want a vehicle that can/could almost fly by itself, so as to achieve, as a minimum, 'automobile-like' levels of ease of use. Autonomous system technology developed for small autonomous rotary-wing vehicles will enable technology transfer to personal transport vehicles.

Planetary Science --

The next few years promise a unique convergence of NASA aeronautics and space programs. NASA planetary science missions are becoming increasingly more sophisticated. This will ultimately culminate, in part, in the development of planetary aerial vehicles. Autonomous vertical lift vehicles hold considerable potential for supporting planetary science and exploration missions (Fig. 1).

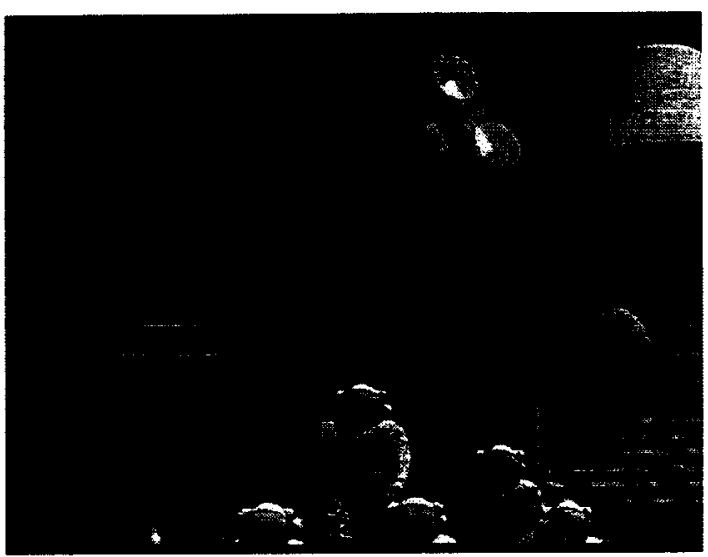

Fig. 1 - Small Autonomous Rotorcraft for Planetary Science Applications

Public Service --

Police and emergency response actions in near-gridlocked urban areas dictates alternate approaches to fast effective public service. Use of autonomous rotorcraft is one approach to address this important issue. In particular, small autonomous rotorcraft (micro air vehicle type) have considerable potential for public service environmental-sensing and police/fire-fighting surveillance (Fig. 2). 


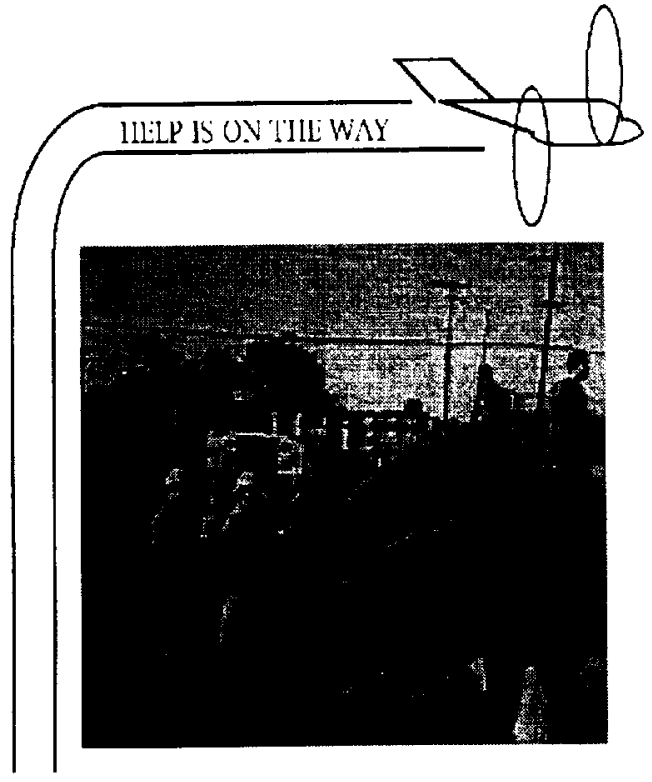

Fig. 2 - Public Service Missions for Small Autonomous Rotary-Wing Vehicles

Small autonomous rotorcraft will afford an exciting opportunity for technical innovation into new areas of vehicle design, rotary-wing aeromechanics, and autonomous system capabilities. Tried and true design approaches for conventional rotorcraft can and must be set aside to examine this emerging new vehicle class.

From a functionality perspective, rotarywing micro air vehicles can be seen to fill three general categories: covert surveillance applications; mobile user interaction and utility functions; and implementation of rapid deployment of 'distributed' processes.

\section{Covert Surveillance:}

Covert surveillance applications benefit from the inherent low observability of rotary-wing micro air vehicles. Distributed (large survey areas with a large number of sensors) missions and applications can benefit from "swarms" of very small vehicles operating in a coordinated, costeffective, and efficient manner. Mobility enhances the flexibility of intelligence gathering. Use of multiple distributed vehicles minimizes the impact of individual vehicle detection/compromise to the overall covert operation. Small vehicles are potentially easier to insert into target territory unobtrusively. For example, small vehicle size could enable manual transport/fielding by one or two soldiers/operators. Alternatively, very small, lightweight, and robust vehicles could be dispersed from a 'mothership' aerial platform in a similar manner as aerosols, cluster-type munitions, or psychological operations leaflets or other materials.

Mobile User Interaction and Utility Function:

It is currently not unusual for people to carry one or more digital electronic devices for day-to-day personal or professional. The future promises an explosion of personal digital devices. Even wearable computers are seriously being studied. Use of micro-rotorcraft for personal interactive services is a natural logical extrapolation of this trend. Micro-rotorcraft in close proximity to and interacting with people could provide unprecedented personal utility and function. "Distributed" processing/sensing across multiple vehicles could provide enhanced utility without increasing any individual vehicle's weight or size. Further, vehicle mobility would reduce, or eliminate, the manual 
burden of transporting this functionality (versus hand-carried or wearable devices). For example, rotary-wing micro air vehicles could be used as flying 'personal' video cameras for high-end consumers/world-travelers. Other examples: improved Secret Service protection for high-level politicians/dignitaries; improved management of prisoner release programs; personal security; achievement of true secure communication through actual physical transfer of information by means of micro-rotorcraft acting as 'couriers.'

\section{Rapid Deployment of Distributed} Processes:

Large areas can be surveyed or secured more quickly and comprehensively with multiple small aerial vehicles carrying multiple types of sensors and/or active devices. This task can be accomplished most effectively with small, low-cost vehicles that can be rapidly and easily dispersed, and are considered ultimately to be expendable. Examples could include: chemical spill surveys and clean-ups (timed release of petrochemical-eating bacteria as one example); surveillance for treaty violations of weapons of mass destruction (chemical, biological, and nuclear); facility or building physical security; border security; planetary exploration; revealing, documenting, and perhaps preventing potential crimes against humanity (via rapid insertion of active, intrusive, pervasive, and visible intelligence assets primarily comprised of microrotorcraft).
There are at least two fundamentally different perspectives related to the development of rotary-wing micro air vehicles. One perspective, or research focus, seems to be more about pushing the frontiers of micro-machine technology rather than being mission or application driven. The mission/application focus is just now gaining visibility. This is the perspective that the Army/NASA Rotorcraft Division has been emphasizing in its research studies. Further, the Rotorcraft Division has also emphasized vehicle design innovation in its micro-rotorcraft research - making the fundamental assumption that mission requirements for very small rotary-wing platforms with autonomous system capability will drive the development of radically new vehicle configurations -see Fig. 3. However, at this early stage of investigation, both technical approaches -- technology-driven versus mission/application-driven - should continue be pursued in parallel. It is likely, ultimately though, that missiondriven vehicle research and technology will focus on a wide-spectrum of vehicle scale (ranging from MAV-class vehicles and current rotorcraft UAVs (for example, the Sikorsky Cypher and the Bell Eagle-Eye). 

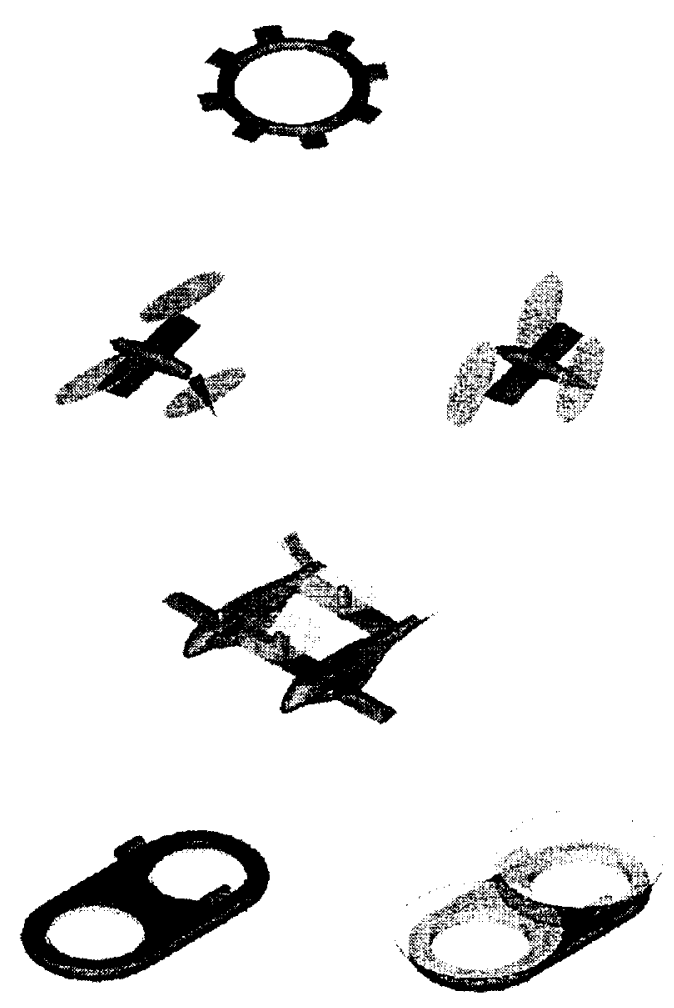

Fig. 3 -- Various Notional MicroRotorcraft Concepts

Table 1 is a matrix of various missions deemed appropriate for certain ranges of vehicle size. Though there is some subjectivity as to the classes of vehicle size selected, and some room for argument for whether a particular vehicle size can perform a given mission, this table hopefully provides an initial starting point for subsequent micro-rotorcraft and small autonomous rotary-wing vehicle mission and application discussion.
Table 1 -- Vehicle Size versus Types of Mission

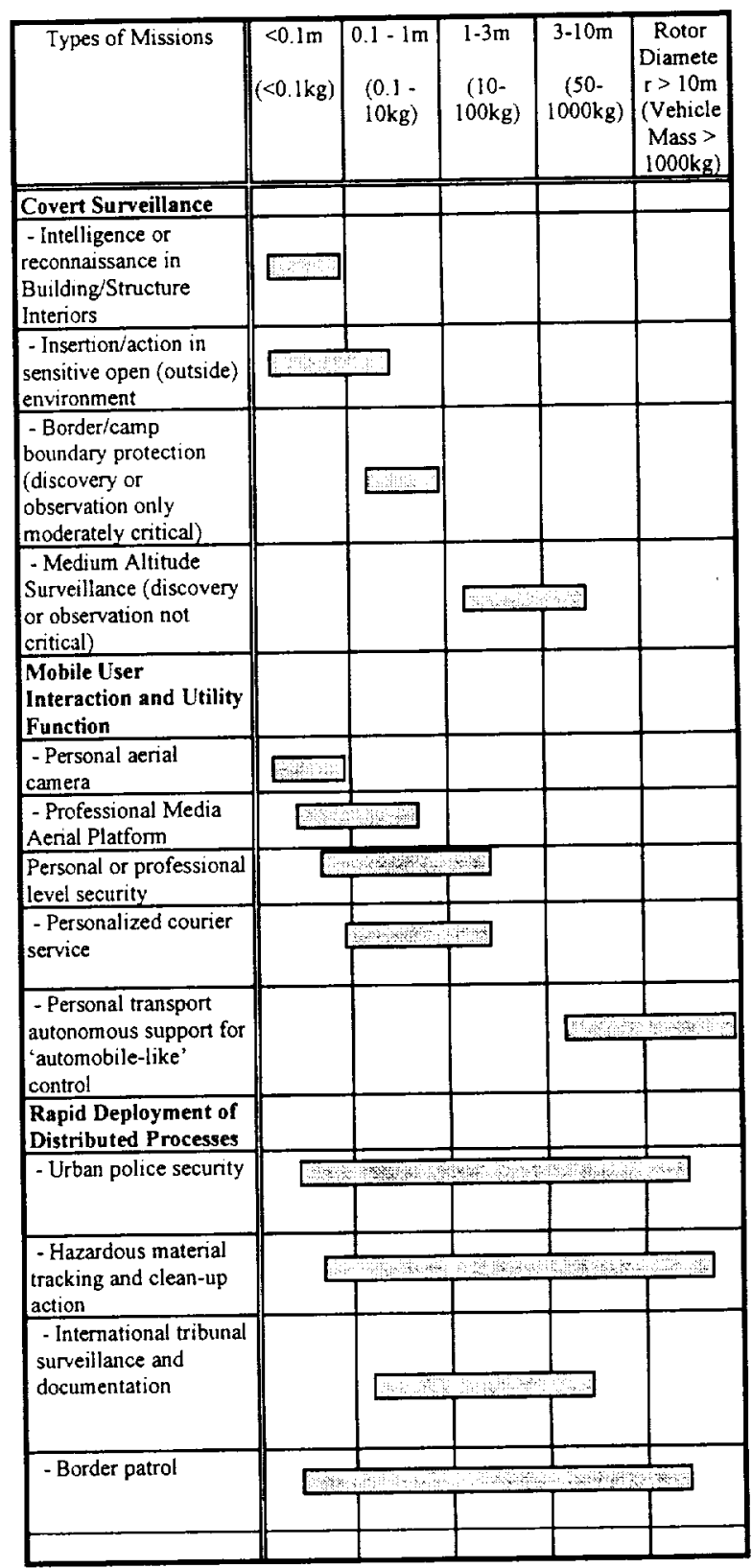

It is important to ask what are the technical issues, and limitations, of 'micro-rotorcraft?' Low Reynolds number aerodynamics and low aspect ratio lifting surfaces will be a challenge in that limited data exists for these flow regimes and aerodynamic configurations. Further, there is little 
information/insight into the rigorous design of these very small vehicles. The closest analog to micro-rotorcraft are hobbyist, or radio-controlled, helicopters. But it is important to note that small autonomous rotorcraft capabilities will need to go well beyond 'hobbyist' or 'industrial' radio-controlled helicopter capabilities. Nonetheless, there perhaps can be some lessons learned from the hobbyist world that can be applied to small autonomous rotorcraft development.

Another challenge will be providing adequate onboard resources to perform practical missions/application (including providing adequate fuel/power to fly reasonable ranges and endurance, adequate payload fraction for mission package for sensors and downlink telemetry/transmission, high-speed, multiprocessor flight/mission computer architectures compatible with both lowand high-levels of autonomy.

Low-cost manufacturability will be essential to rotary-wing micro air vehicles (a large number of these vehicles will be required as compared to larger vehicles, and these very small rotorcraft vehicles will essentially be 'disposable'). Minimum man-in-theloop effort is also essential for operating and interrogating these very small (and potentially large numbers of) rotorcraft vehicles. Vehicle endurance, particularly for vehicles powered by electric propulsion, will be a major issue. Current state of the art motor and battery technology limits electric-powered hobbyist helicopters less than a half-hour of flight at best. Endurance limitations of individual small autonomous rotorcraft can be compensated for by using multiple platforms to accomplish a given mission in that a constant cycle of vehicles could either be 'recharging' themselves or performing the station keeping mission. As these vehicles are very small and low-cost such a constant re-supply/mission sortie cycle should be viable for even simple missions. One possibility to expedite such a constant re-supply/mission sortie cycle strategy would entail having a 'perch' for the vehicles which would incorporate an induction-plate electrical charger. Another possibility is to have the vehicles drop to the ground and recharge via solar cells before returning to flight and the mission.

Powerful market and technological forces will help influence the successful development of small autonomous rotorcraft and micro-rotorcraft. The fastpaced world of consumer electronic/digital products is driving the development of new advanced battery and/or fuel-cell technologies, micromechanical sensors, high-speed, lowpower, distributed micro-processors, and other miniature electronic components necessary for small autonomous rotorcraft. Finally, there has been a renaissance recently as to automated reasoning and robotics. NASA research into information technology and intelligent systems research has seen significant increases in the past few years. NASA has a long-term strategic goal to developed robotic systems ostensibly for space and planetary science missions but with potential broad application - including autonomous rotorcraft. Further, this NASA strategic goal includes studies into distributed robotic 'colonies' with multiple, and heterogeneous groups of, 'agents.' This work is being performed inhouse and through NASA sponsorship 
by industry and academia. All of this work in automated reasoning, robotics, robotic colonies, and distributed processing will have significant implications for the successful introduction of autonomous rotorcraft.

\section{Ames Design and Technology Efforts}

The work to date at NASA Ames has focused on the usage of electric propulsion for the micro-rotorcraft proof-of-concept vehicles. This has been an intentional programmatic decision on the part of the microrotorcraft team. The use of electric propulsion enforces a crucial vehicle development discipline with respect to vehicle aerodynamic efficiency, ultralightweight structures, and efficient drive train and actuator components.

To date a number of micro-rotorcraft concepts have been taken to an initial proof-of-concept test article stage. These vehicles and their associated proof-of-concept testing will be briefly discussed in next section and more extensively in the final paper.

\section{Quad-Rotor Tail-Sitter}

Figure 4 is a photograph of a quad-rotor tail-sitter concept. Quad-rotor designs employing rotor speed control for vehicle trim yields a very simple but very effective control system approach. The simplicity of rotor speed control lends itself well to micro-rotorcraft applications where packaging and providing adequate control power for very small vehicles becomes extremely difficult. Combining a flying-wing, tailsitter design with quad-rotor propulsion potentially results in a vehicle that has full hover capability but with high-speed aerodynamic efficiency that have significant range and endurance capability. Employing a flying wing in the design also allows for the possible integration of solar-cell arrays on the wing surface, and thereby enabling longterm, extended-duration (on the ground) missions. The limited wing area inherent in a small vehicle would dictate that the vehicle would have to recharge on the ground, between flights, but would allow several flights over several days, at several different surveillance sites.

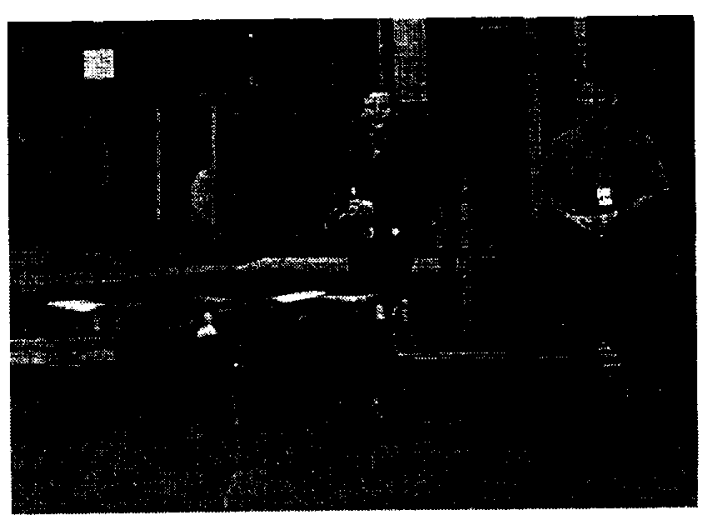

Fig. 4 -Quad-Rotor Tailsitter in Hover Mode

Tailsitter designs have had a mixed history as to their successful demonstration. However, many of these developmental problems and limitations were a consequence of the flight demonstrators being inhabited/piloted aircraft. UAV applications for tailsitter designs would likely yield acceptable vehicle configurations. 
Controlled hover and low-speed flight has been successfully demonstrated to date for the quad-rotor tailsitter design. Forward-flight transition has also been demonstrated. Further vehicle improvements are required before demonstrating sustained controlled forward-flight.

\section{'Mini-Morpher'}

Micro-rotorcraft missions will likely require these vehicles to fly at lowaltitudes, in the close presence of people and buildings. Such close proximity with objects to potentially collide with will dictate that micro-rotorcraft have as small of a mass (and impact momentum) as possible, while still meeting overall mission requirement -- thereby minimizing the potential for damage to the vehicle and it surroundings in case of unavoidable collisions. Further, this same concern regarding low-altitude collision with people and things will foster the development of vehicles that in some manner take steps to physically protect their rotors and other critical hardware from accidental impact damage. This is why, for example, there is considerable recent interest in ductedfan versions of vertical lift micro air vehicles.

Recent improvements in active flight controls and smart materials/actuators now make possible low-mass micromechanical systems that allow microrotorcraft designs that can "morph," i.e. modify vehicle geometry to match flight condition.

NASA Ames has developed some ideas related to the use of morphing vehicle technology to "shroud" micro-rotorcraft rotors while in hover and low-speed flight, and then change configuration in high-speed forward flight to maximize aerodynamic efficiency. One such concept is the "mini-morpher" - see Fig. $5 a-b$.
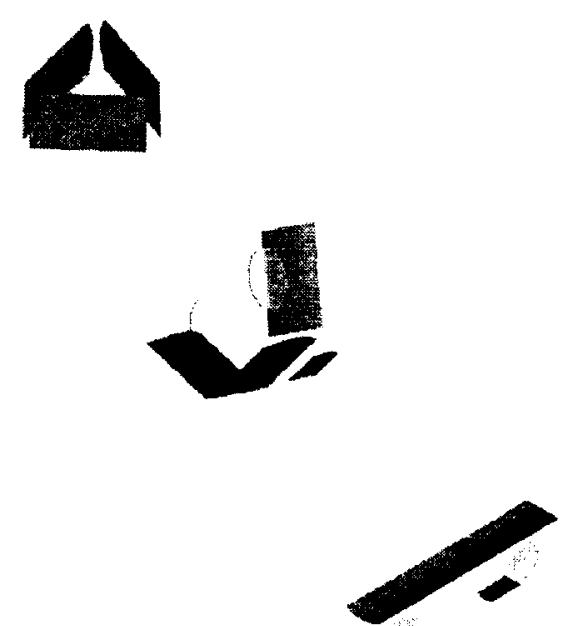

(a)
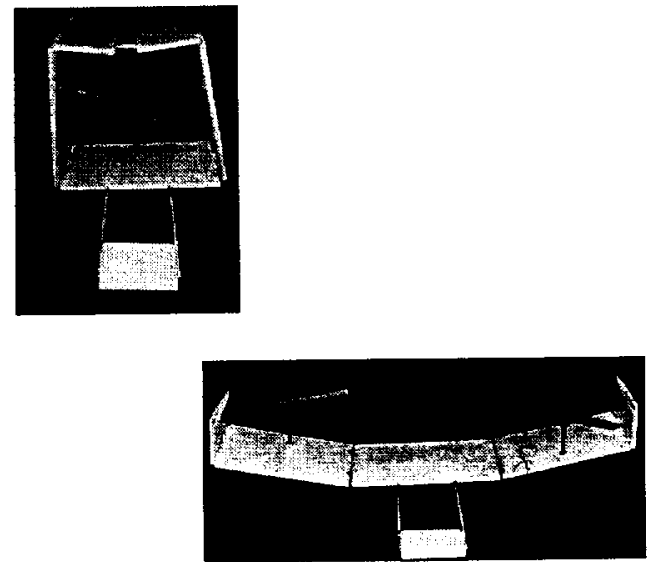

(b)

Fig. 5 -- 'Mini-Morpher' Transitioning from Hover (Top Left) to ForwardFlight (Bottom Right): (a) Concept and (b) Prototype/Test Article

Tethered hover testing has been demonstrated to date with the minimorpher test articles. 


\section{Twin-Fuselage Tandem Tiltrotor}

The pursuit of efficient forward flight characteristics while preserving good hover performance is the goal for all rotary-wing vehicles. However, to pursue this goal while at the same time minimizing overall vehicle size and the potential for low-altitude (from ground level to a couple hundred feet at most) collision damage to the vehicle and people/surroundings makes for a unique design challenge.

The conventional tiltrotor aircraft configuration (as represented by the XV15 and V-22 aircraft) meets the prerequisite requirements for efficient hover and forward-flight. However, because the conventional tiltrotor aircraft's rotor and engine nacelles are mounted on the vehicle's wing-tips, micro-rotorcraft versions of these vehicles would be especially prone to low-altitude collision damage with people and objects (as well as possible landing on rough ground/vegetation). An alternate tiltrotor/tiltwing configuration is being considered within NASA Ames: a twin-fuselage, tandem tiltrotor (Fig. 6a-b). The twin fuselages of this vehicle protects its rotors from accidental impact/collision damage by nestling the rotors between the fuselage airframes.

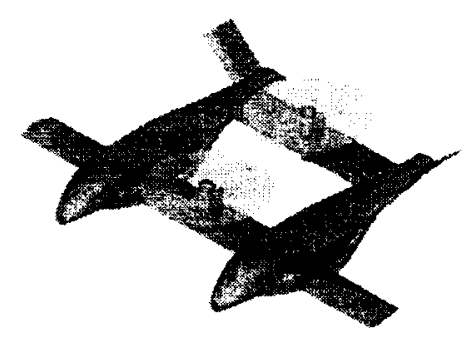

(a)

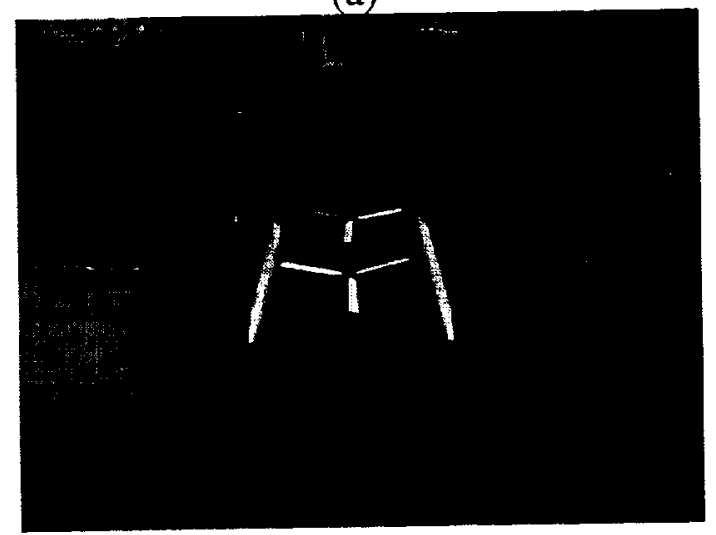

(b)

Fig. 6 - Twin-Fuselage Tandem Tiltrotor: (a) Concept and (b) Prototype

\section{Coaxial Helicopter}

Coaxial helicopters have been successfully flying for several decades now - including a series of UAV or RPV platforms (the Candair CL-237, the Westland 'Sprite,' and the Gyrodyne QH-50, among others). Even a few custom-designed coaxial hobbyist RC models have been flown. These coaxial helicopter UAVs are fairly complex mechanical systems. Implementation of such complex mechanical systems becomes ever increasingly difficult as the scale of the vehicle is reduced.

An alternate, simpler, coaxial helicopter configuration is being studied by NASA Ames (Fig. 7a-b). By employing two fully symmetric (but mirrored -- with the 
exception of the rotors -- hardware) drive train, control system, and rotors a simple, minimum complexity coaxial helicopter configuration has been developed. A full-span (across the rotor disk) cross-brace/spar between the two rotors supports the two independent drive trains. Vehicle yaw control is effected by differential torque between the two drive trains resulting from a differential collective setting for the two rotors.

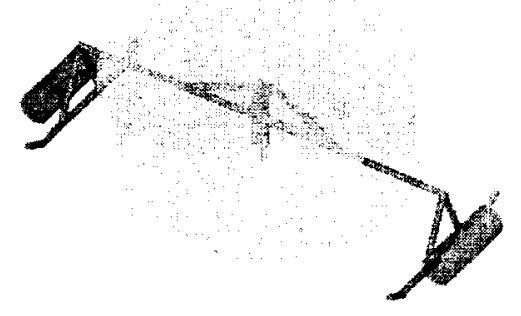

(a)

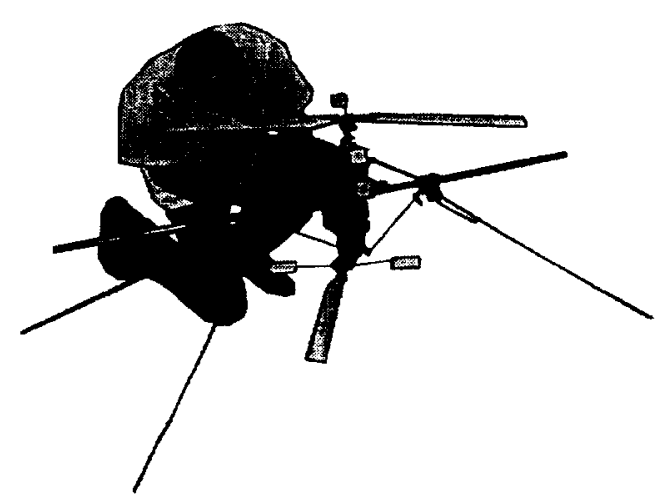

(b)

Fig. 7 - Reduced Complexity Coaxial Helicopter: (a) Concept and (b) Prototype

The intent of the proposed paper is not to identify the 'best' micro-rotorcraft design from a field of prospective candidates. Instead, the objective of the final paper will be to suggest that considerable opportunities still exist to think 'outside the box' and consider wholly new vehicle configurations for micro-rotorcraft and other small autonomous rotary-wing vehicles. It would be extremely disappointing - and ultimately self-defeating -- if only 'scaled down' versions of existing, conventional inhabited/piloted rotorcraft types were developed for microrotorcraft applications.

\section{General Micro-Rotorcraft Hover Performance Characteristics}

Rotor performance is a crucial aspect of micro-rotorcraft and small autonomous rotary-wing vehicle design. For example, low Reynolds flow (1000 to $10,000)$ on rotor blade sections can result in comparatively high airfoil profile drag.

In addition to the inhouse work at NASA Ames focusing on vehicle concept development, a complementary effort is being conducted examining rotor performance characteristics for very small rotor systems. These hover performance experiments are conducted with fairly simple test apparatus. A series of experimental measurements of hover performance for a variety of rotor parameters for rotors of the approximate scale of micro-rotorcraft configurations is being conducted and will be reported in the final paper.

\section{Test Description}

To measure the thrust produced by very small rotors in hover, a lever balance system was constructed (Fig. 8). The 
force output from the lever system was applied by knife edge to a commercial digital scale. Rotor power measurements were made with a wattmeter, and a correction methodology was developed to account for not only hub tares but also to measure and apply corrections for the stand drivetrain/motor efficiencies.

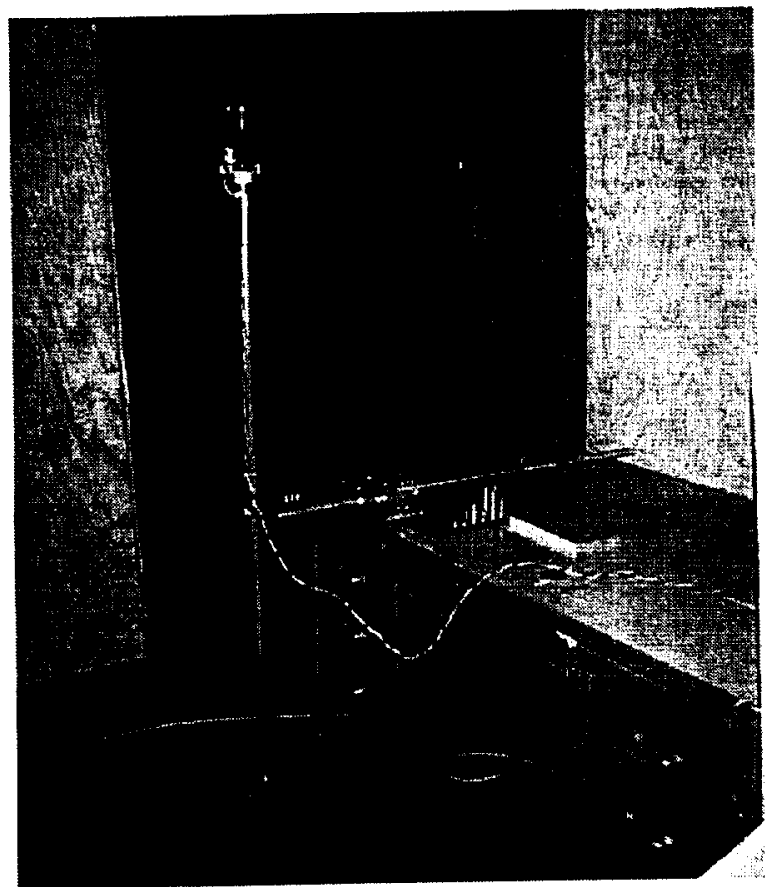

Fig 8 - Hover Test Stand

In order to raise the rotor out of ground effect, the rotor was mounted on top a long slender support strut. Minimum wake blockage results from the compact stand design. Weights were added to both sides of the lever/balance system to both minimize the digital scale zerooffset as well as creating a large moment of inertia in the system to minimize large unsteady rotor/wake oscillations during testing.
Different rotors are to be tested with several different rotor blade solidity values and other blade geometry variations. A brief description of the baseline rotor tested is given in Table 2 and shown in Fig. 9. Prior to hover testing the blade pitch angles were manually set. RPM was varied remotely by a radio transmitter. Rotor speed was measured by a digital tachometer, which was positioned on a swing arm that could move into and out of the rotor wake underneath the rotor so as to trigger off of the blade tip passage. All rotor performance measurements were made with this tachometer/swing-arm out of the rotor wake flow.

Test safety shielding was sufficiently removed in distance from the rotor to ensure little wall effect on rotor performance. All safety shielding was tested to ensure that any flying debris would not harm personnel in the test area.

Table 2 -- Baseline Rotor Description

Rotor hub
Stock electric radio-
controlled (RC) helicopter
hub
Collective (blade pitch
angle): manually adjusted
Cyclic: servo controlled, but
fixed at zero angle for testing
Hub Type: teetering hub with
Hiller flybar
Blade Grip Radius: $5.5 \mathrm{~cm}$




Rotor Blades
Number of blades: 2
Airfoil Type: flat plate with
circular arc camber,
approximately 12\% chord at
$50 \%$ chord station
Twist: linear, -10 degrees
Solidity: 0.0560
Taper Ratio: .449
Aspect Ratio: 7.283
Blade Length (measured
from grip location to tip): 25
cm
Blade Radius (including
blade grip radius): $30.5 \mathrm{~cm}$
Motor Assembly
Motor: fractional horsepower
electric motor
Single Stage Gear Reduction
Ratio: $12: 1$

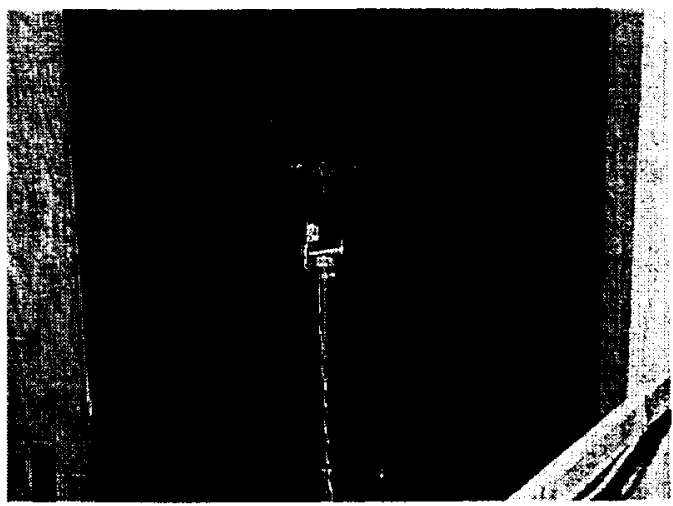

Fig. 9 -- Baseline Rotor

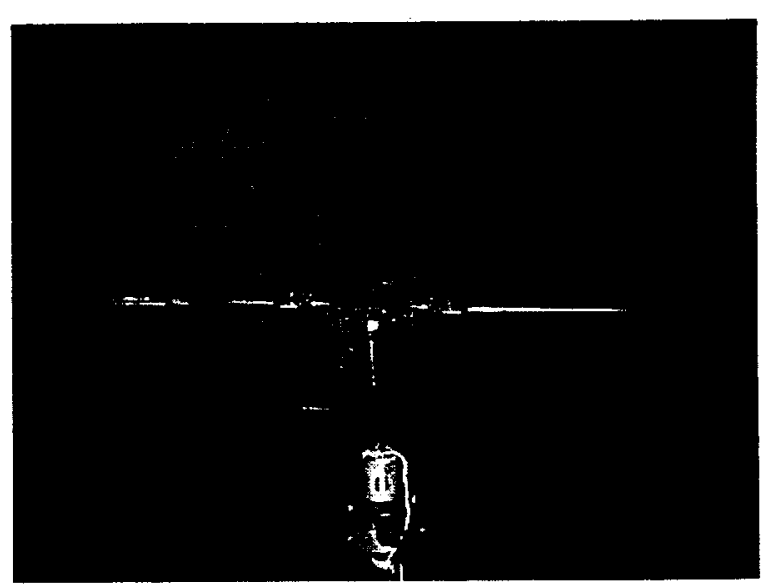

Fig 10 - Rotor Bat Testing

\section{Experimental Results}

Results from rotor bat tests were used to calculate a motor efficiency factor (Fig. 10). The rotor bats tested were cylindrical in cross section and of constant diameter across the span of the rotor bat. The rotor bat tests showed through data acquired from testing several different rotor bats of varying length -- that the motor efficiency was essentially independent of loading. The trend of motor efficiency with speed for the hover test stand drive motor is given in Fig. 11.

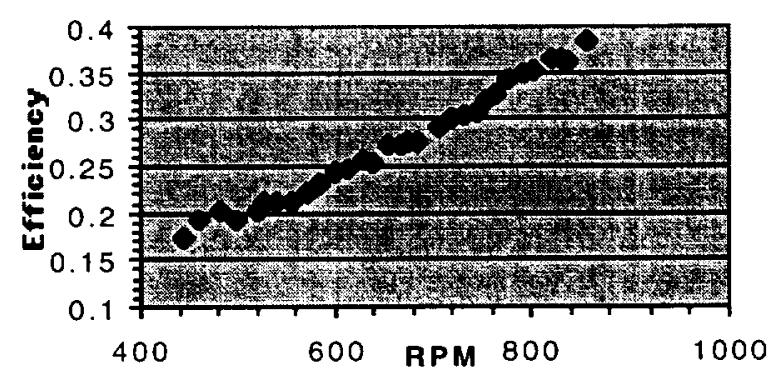

Fig. 11 - Motor Efficiency Versus Speed 
Measured motor efficiencies ranged from 0.12 to 0.38 . A nominal factor of 0.285 was selected to multiply to the motor input power data to yield an estimate of rotor-shaft power. This approach for deriving rotor-shaft was quite successful for the testing of microrotorcraft-class rotors.

Figure 12 shows the measured figure-ofmerit for the baseline rotor at the nominal RPM, varying blade pitch angles (rotor speed was approximately 605 RPM, with a resulting tip Mach of approximately 0.057 ).

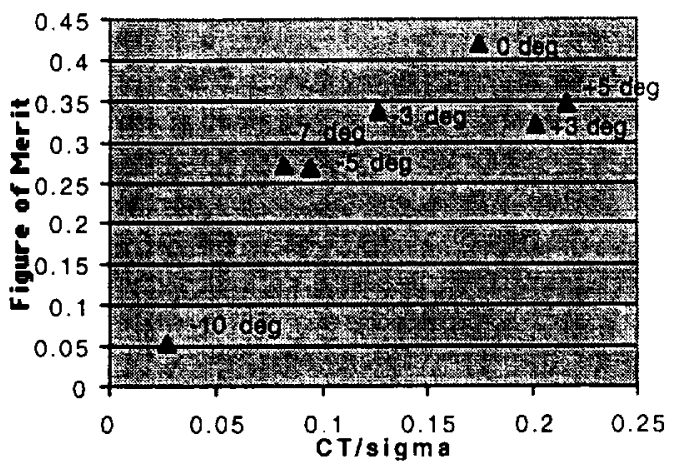

Fig. 12 - Baseline Rotor Figure-of-Merit as a Function of Thrust

The overall figure-of-merit trend for the small baseline rotor is similar to that seen for large, conventional rotorcraft -though the magnitude of the figure-ofmerit values is comparatively very low. These low figure-of-merit values for the small baseline rotor are consequence of high profile power, which in turn is due to the high profile drag coefficients typical for airfoils in this particular (low) range of Reynolds number. Similar test results have been previously reported (Ref. 2). The onset of rotor stall is clearly seen in the baseline rotor figure- of-merit curve for the $C_{T} / \sigma$ range of 0.15 to 0.2 .

Figure 13 shows the $C_{p} / \sigma$ versus $C_{T} / \sigma$ power-polar for the same RPM, once again varying collective. Again the general trend of the power-polar is similar to large conventional rotorcraft, except that the $C_{p} / \sigma$ values are comparatively much larger than typically seen for conventional rotorcraft.

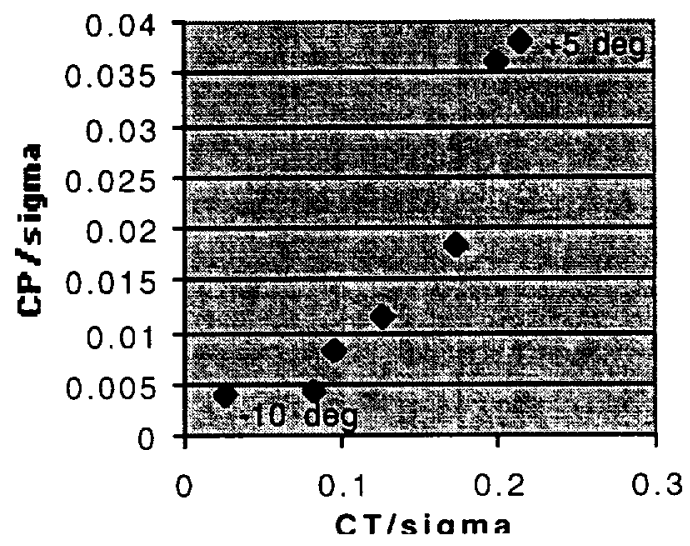

Fig. 13 - Baseline Rotor Power Polar Curve

The data for $\mathrm{C}_{\mathrm{T}} / \sigma$ versus the blade pitch angle theta (measured at the $18 \%$ radial station) is shown in Fig. 14. This approximately linear trend is also similar to that seen for larger rotorcraft. 


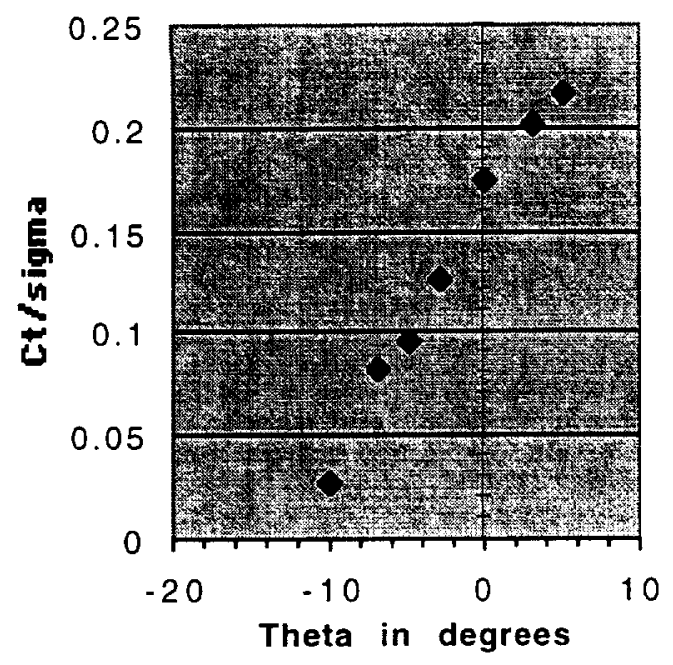

Fig. 14 - Baseline Rotor Thrust Versus Collective

The final paper will report on hover test results for other micro-rotorcraft-class rotors as well. In particular, the influence of parametric rotor geometry changes on rotor performance will be closely examined (Fig. 15).

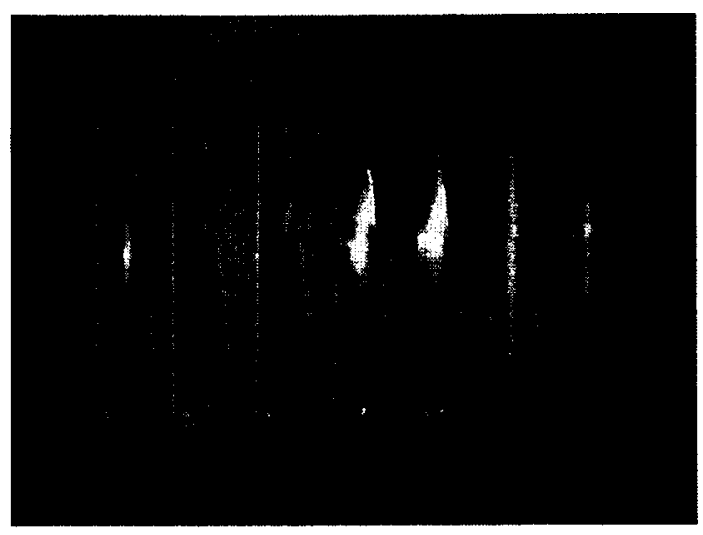

Fig. 15 - Parametric Investigation of Rotor Solidity on Hover Performance

\section{Micro-Rotorcraft Weight Trends}

Existing preliminary design (PD) tools are incompatible with micro-rotorcraft applications. In particular, weight trend data and PD weight equations are not scaleable to these very small vehicle sizes. What the rotorcraft community requires is a set of $\mathrm{PD}$ tools that is broadly applicable across the spectrum of the rotary-wing vehicles which includes not only conventional (inhabited) rotorcraft but rotorcraft UAVs, small autonomous rotary-wing vehicles, and micro-rotorcraft. As an incremental step towards developing such cross-spectrum design tools, a data compilation of $\mathrm{RC} /$ hobbyist hardware component weights (fixed- and rotarywing) for a variety of vehicles has been performed and modified PD weight estimate equations are being developed that will be scaleable across the widespectrum of rotary-wing vehicles, including micro-rotorcraft. The functional form of these equations will originate from standard rotorcraft PD weight equations (see Refs. 3-8) but will be, by necessity, modified to accommodate the smaller vehicle weight trend data. To accomplish this goal measurements were made for various component/sub-system weights for radio-controlled electric helicopters (Figs. 16 and 17). These component weights are then used to refine/modify the semi-empirical weight trend equations used by the rotorcraft preliminary design community. The results of this effort will be reported in the final paper. The modified weight equations should enable an improved design process for future microrotorcraft development efforts. 


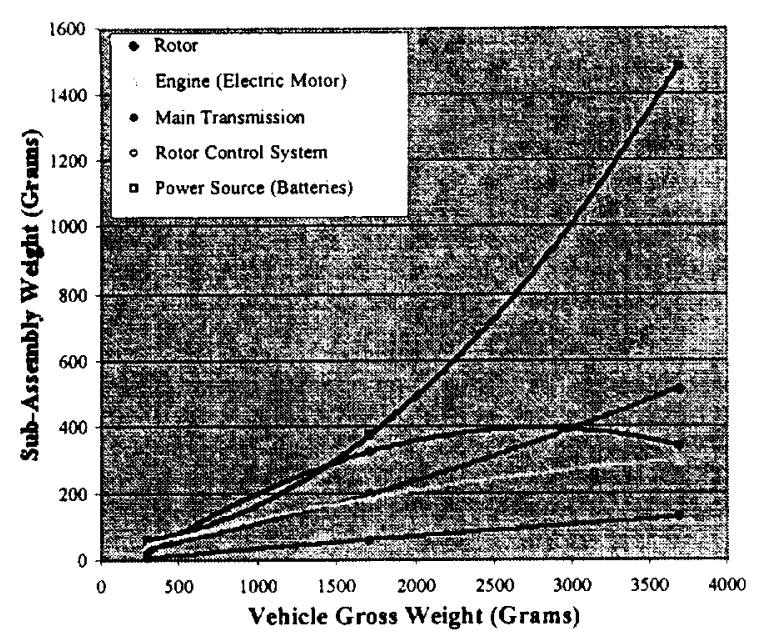

Fig. 16 - Rotor/Propulsion System

Weights

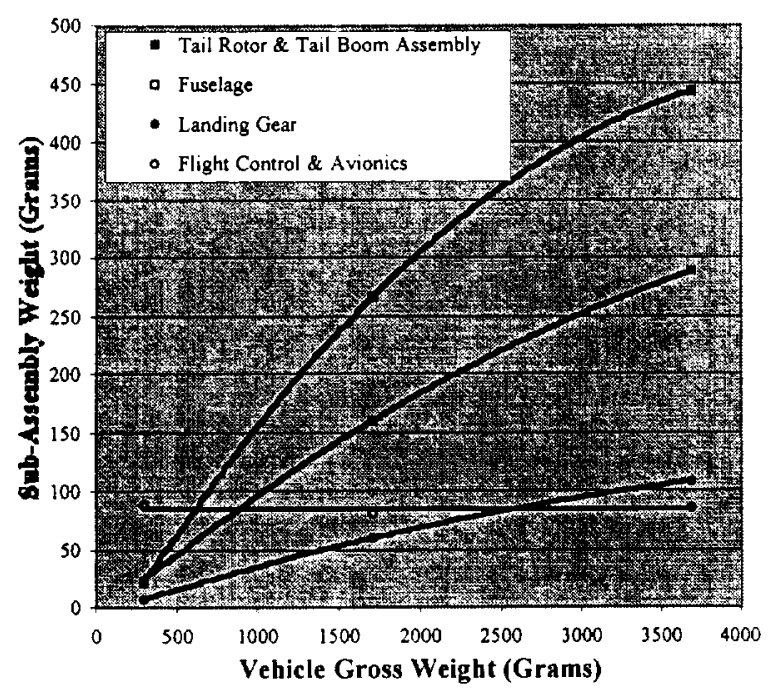

Fig. 17 - Airframe Component Weights

\section{Future Work}

The Army/NASA Rotorcraft Division at NASA Ames Research Center continues to develop new vehicle concepts for micro-rotorcraft applications. As concepts are matured beyond the proofof-concept phase then issues of automated flight control will be examined and implemented from a research perspective. The ultimate goal of the micro-rotorcraft project is the development of a suite of concepts and technologies that will enable a new class of flight vehicles to enter into practical application.

Finally, one of the more significant merits of the NASA Ames microrotorcraft project is the opportunity to propose and examine innovative vehicle concepts that could not be costeffectively evaluated at larger scales.

In parallel to the micro-rotorcraft work within the Army/NASA Rotorcraft Division, research investments are being made in the area of autonomous systems technology for rotorcraft. It is hoped that cross-platform technology can be developed such that high-levels of vehicle autonomy can be ported to a wide array of rotary-wing vehicles, including micro-rotorcraft.

\section{Concluding Remarks}

Three noteworthy accomplishments have been made and will be reported in the final paper as related to the development and understanding of micro-rotorcraft and small autonomous rotary-wing vehicles: 1. A series of innovative vehicle concepts suitable for application to micro-rotorcraft and small autonomous rotorcraft application have been proposed and studied with respect to establishing initial proof-of-concept; 2. A general assessment of the hover 
performance characteristics of low Reynolds rotors has been experimentally evaluated; 3 . Weight trend equations have been developed that bridge the gap between preliminary design application for micro-rotorcraft and conventional rotary-wing flight vehicles. As a consequence, the results of the final paper will hopefully inspire follow-on work to fully realize the potential benefits of micro-rotorcraft and small autonomous rotary-wing vehicles for our society.

\section{Acknowledgement}

The work summarized in this paper was supported by discretionary funding from the Aerospace Directorate at NASA Ames Research Center. The contribution of Ms. Naomi Tsafnat to the micro-rotorcraft project is also gratefully acknowledged.

\section{References}

1. Aiken, E.W., Ormiston, R.A., and Young, L.A., "Future Directions in Rotorcraft Technology at Ames Research Center," 56 ${ }^{\text {th }}$ Annual Forum of the American Helicopter Society, International, Virginia Beach, VA, May 2-4, 2000.

2. Samuals, "MICOR," AHS Vertical Lift Aircraft Design Conference, San Francisco, CA, January 2000.

3. Stepniewski, W.Z. and Keys, C.N. , Rotary-Wing Aerodynamics, Dover Publications, Mineola, NY, 1984.
4. Davis, A.J. and Wisniewski, J.S., "User's Manual for HESCOMP: The Helicopter Sizing and Performance Computer Program," NASA CR 152018, September 1973.

5. Stepniewski, W.Z. and Shinn, R.A., "Soviet Vs. U.S. Helicopter Weight Prediction Methods," $39^{\text {th }}$ Annual Forum of the American Helicopter Society, St. Louis, MO, May 9-11, 1983.

6. Stepniewski, W.Z., "Some Weight Aspects of Soviet Helicopters," $40^{\text {th }}$ Annual Forum of the American Helicopter Society, Arlington, VA, May 16-18, 1984.

7. Vega, E., "Advanced Technology Impacts on Rotorcraft Weight," $40^{\text {th }}$ Annual Forum of the American Helicopter Society, Arlington, VA, May 16-18, 1984.

8. Smith, H.G., "Helicopter Structural Weight Prediction and Evaluation Theory Versus Statistics," $26^{\text {th }}$ Annual Forum of the American Helicopter Society, Washington, DC, June 16-18, 1970. 